\title{
Prosthetic Rehabilitation of Velopharyngeal Insufficiency with Pharyngeal Obturator Prosthesis: A Case Report
}

\author{
Ashish Thakur ${ }^{1}$, Dushyant Chauhan ${ }^{2}$, RK Yadav $^{3}$, Amit Khattak $^{4}$
}

\begin{abstract}
Soft palate is a part of the palate composed of muscles and mucous membrane. It divides oropharynx from the nasopharynx. Soft palate along with lateral and posterior pharyngeal walls create a three-dimensional muscular valve which is known as velopharyngeal (VP) sphincter. This VP sphincter allows normal breathing and prevents regurgitation of food and fluids in the nasal cavity. It is also required in other functions such as swallowing, blowing, sucking, whistling and sneezing. Any defect in the soft palate, i.e., structurally or neurologically will lead to VP defect due to which normal functions were impaired. Pharyngeal obturator prosthesis restores the congenital and acquired defects of the soft palate and allows adequate closure of palatopharyngeal sphincter. In the present case report, a male patient suffering from pleomorphic adenoma of the soft palate was operated leading to VP insufficiency. This patient was rehabilitated by pharyngeal obturator prosthesis for the improvement of quality of life of the patient.
\end{abstract}

Keywords: Pharyngeal obturator prosthesis, Speech prosthesis, Velopharyngeal insufficiency.

International Journal of Prosthodontics and Restorative Dentistry (2019): 10.5005/jp-journals-10019-1227

\section{INTRODUCTION}

$S_{a}^{\circ}$ oft palate is a part of the palate composed of muscles and mucous membrane. It divides oropharynx from the nasopharynx. Soft palate along with lateral and posterior pharyngeal walls create a three-dimensional muscular valve which is known as VP sphincter. This VP sphincter allows normal breathing and prevents regurgitation of food and fluids in the nasal cavity. It is also required in other functions such as swallowing, blowing, sucking, whistling and sneezing. Any defect in the soft palate, i.e., structurally or neurologically will lead to VP defect due to which normal functions were impaired. ${ }^{1}$

The impaired VP function can be due to insufficiency or incompetency. VP insufficiency referred to the structural loss of some or all anatomical structure of soft palate. While inadequacy referred to the muscular or neurological incapability to achieve complete closure. In certain conditions, a combination of inadequacy and insufficiency exists such as cleft palate. Prosthetic management of VP incompetency is done by using palatal lift prosthesis while VP insufficiency is done by pharyngeal obturator prosthesis. ${ }^{2,3}$

Pharyngeal obturator or VP prosthesis is a removable prosthesis with a posterior extension which separates oropharynx from the nasopharynx. The distal end of the prosthesis acts as an integral part of soft palate and assists in achieving normal VP closure. This case report explains about prosthetic management VP insufficiency by using pharyngeal obturator prosthesis.

\section{Case Report}

A 32 years male serving soldier (Fig. 1A) reported to the department of prosthodontics with the chief complaint of inability to speak clearly and regurgitation of food and fluids from the nose. Medical history showed pleomorphic adenoma of soft palate left side which was operated along with the extraction of 27,10 months back. The surgical repair of the defect was attempted eight months back which failed eventually. General examination showed a moderately built and well-nourished patient who was well oriented to time, place and person. Extraoral examination showed symmetrical facial forms with no deformity associated with the defect. Intraoral examination
1,2 Postgraduate Resident, ${ }^{3} \mathrm{HOD},{ }^{4}$ Classified Specialist

${ }^{1-4}$ Department of Prosthodontics and Crown and Bridge, Army Dental Centre (R\&R), New Delhi, India

Corresponding Author: Ashish Thakur, Postgraduate Resident, Department of Prosthodontics and Crown and Bridge, Army Dental Centre (R\&R), New Delhi, India, e-mail: ashishthakurprostho@gmail. com

How to cite this article: Thakur A, Chauhan D, Yadav RK, Khattak A. Prosthetic Rehabilitation of Velopharyngeal Insufficiency with Pharyngeal Obturator Prosthesis: A Case Report. Int J Prosthodont Restor Dent 2019;9(1):24-28.

Source of support: Nil

Conflict of interest: None

showed a soft palate defect area of $3 \times 2 \mathrm{~cm}$ (Fig. 1B) in dimension extending from the anterior part of the soft palate involving uvula at the posterior end. Preoperative lateral ceph and cone beam computerized tomography (CBCT) (Fig. 2) showed widened airway in the oropharyngeal region. The patient was diagnosed as VP insufficiency secondary to surgery. As failed surgical palatal repair was attempted earlier, so the patient was planned to rehabilitate by a nonsurgical approach using a VP prosthesis. A definitive obturator was planned after 10 months of surgery.

\section{Technique}

For the fabrication of definitive obturator, a primary impression was made by using irreversible hydrocolloid impression material and a primary cast was poured using type II plaster. The primary cast was surveyed, and designing of the framework was done in which complete palatal plate was selected as a maxillary major with combination clasp assembly at 15, 16 and 25, 26 region and cingulum rest at 13,23 region (Fig. $3 \mathrm{~A}$ ). Then rest seat and cingulum rest preparation were done as per the plan (Fig. 3B), and a final impression was made using polyvinylsiloxane impression material of putty and light body consistency. Then a master cast was poured using type IV dental stone and fabrication of cast partial framework was started. In this block, out and duplication of the master cast was

( ) The Author(s). 2019 Open Access This article is distributed under the terms of the Creative Commons Attribution 4.0 International License (https://creativecommons. org/licenses/by-nc/4.0/), which permits unrestricted use, distribution, and non-commercial reproduction in any medium, provided you give appropriate credit to the original author(s) and the source, provide a link to the Creative Commons license, and indicate if changes were made. The Creative Commons Public Domain Dedication waiver (http://creativecommons.org/publicdomain/zero/1.0/) applies to the data made available in this article, unless otherwise stated. 

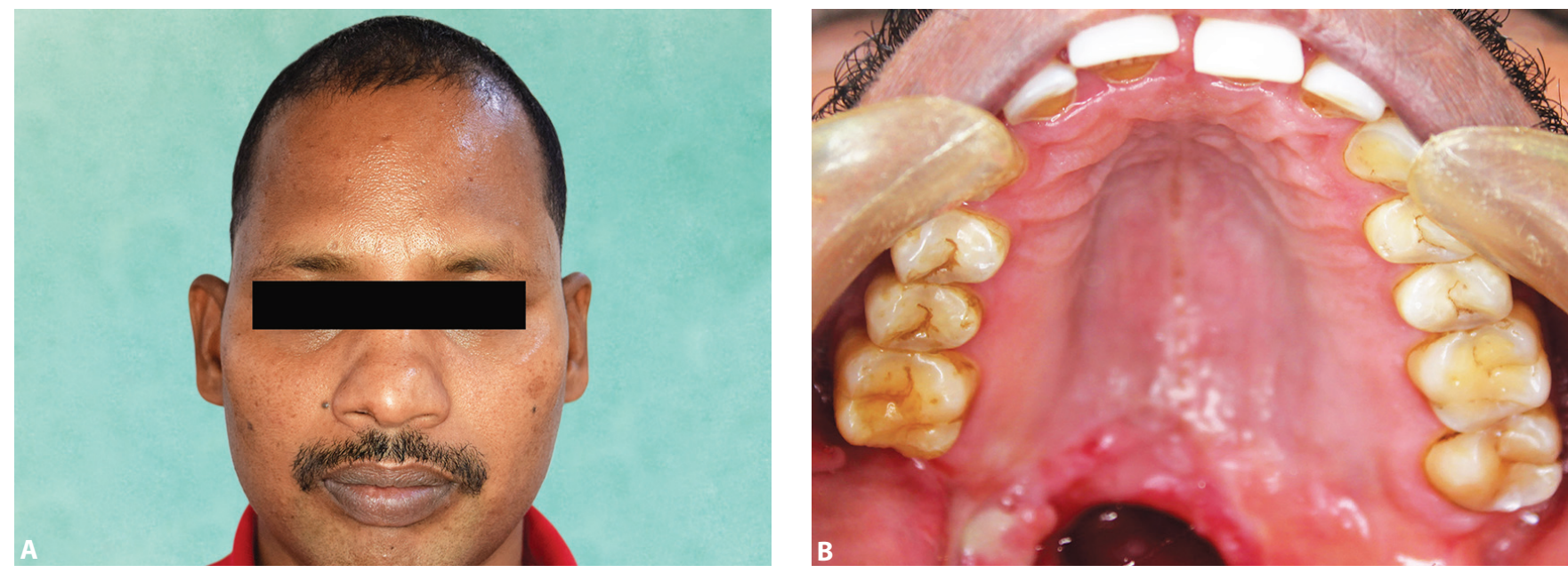

Figs $1 \mathrm{~A}$ and B: Preoperative (A) Extraoral (B) Intraoral
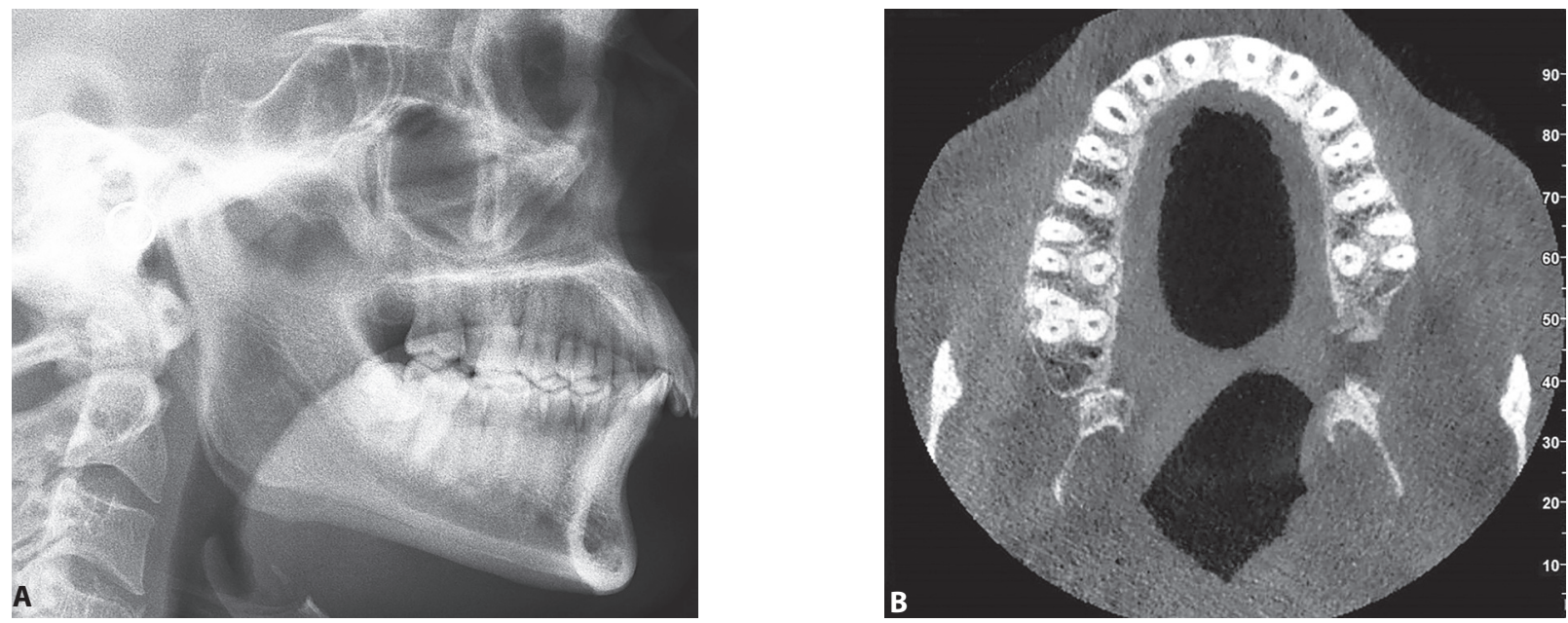

Figs $2 \mathrm{~A}$ and B: Preoperative (A) Lateral ceph (B) CBCT
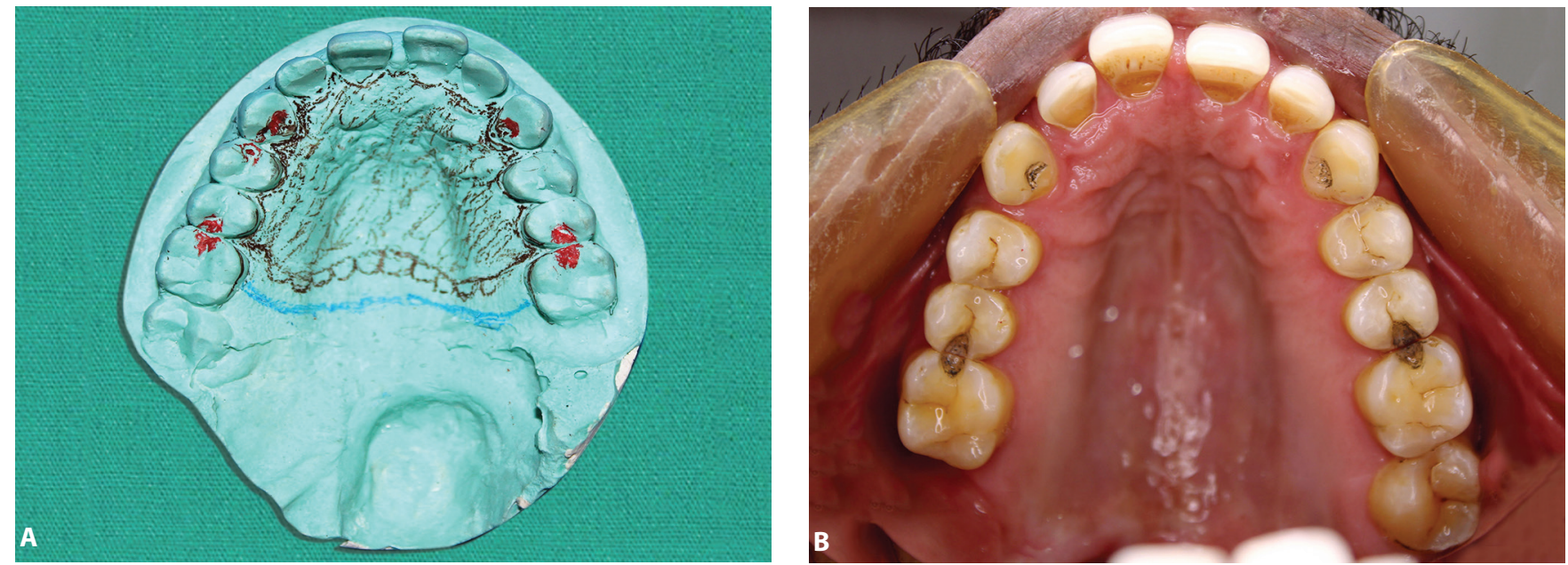

Figs 3 A and B: (A) Designing of master cast (B) Intraoral preparation

done using agar. Then a refractory cast was poured using phosphate bonded investment material followed by wax-up, investment, and casting of the final framework. After finishing and polishing of the cast framework, try in of the framework (Fig. 4A) was done to check for the fit and accuracy of the final prosthesis. Then a 21 gauge orthodontic wire was bent and soldered at the distal end of the framework (Fig. 4B). With the wire framework border molding of the defected area was done using low fusing impression 

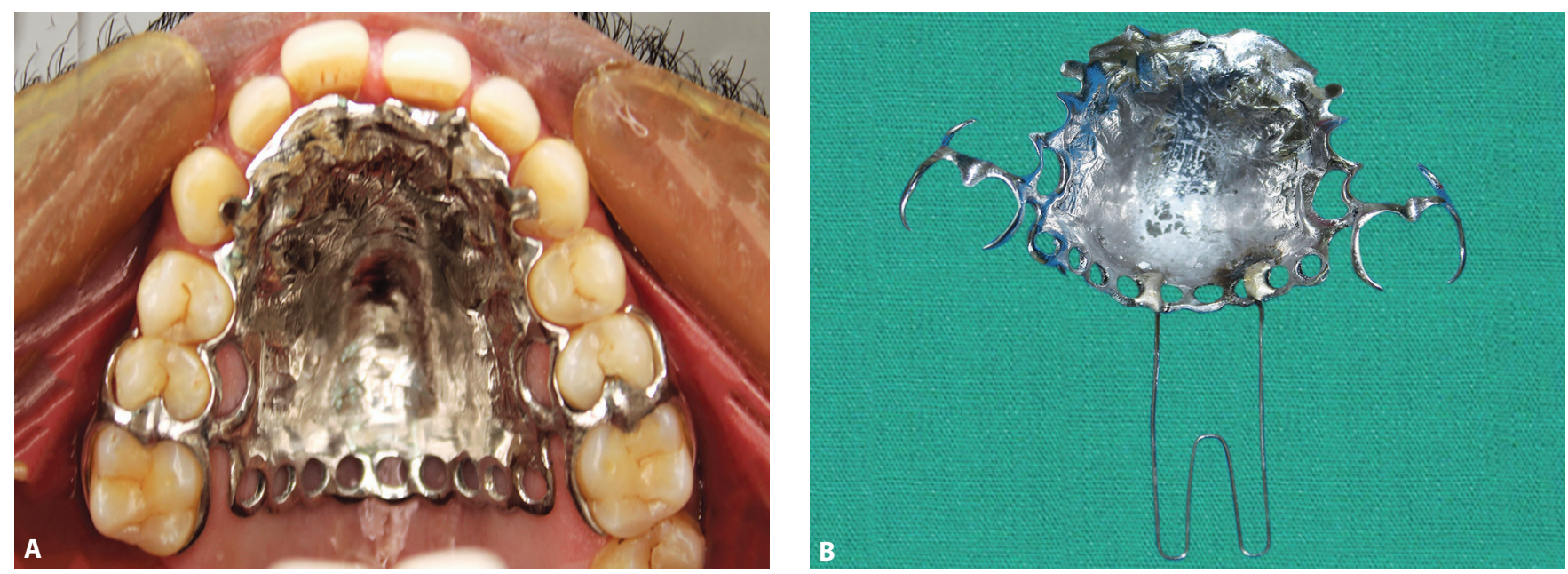

Figs 4A and B: (A) Framework try in (B) Soldered wire extension
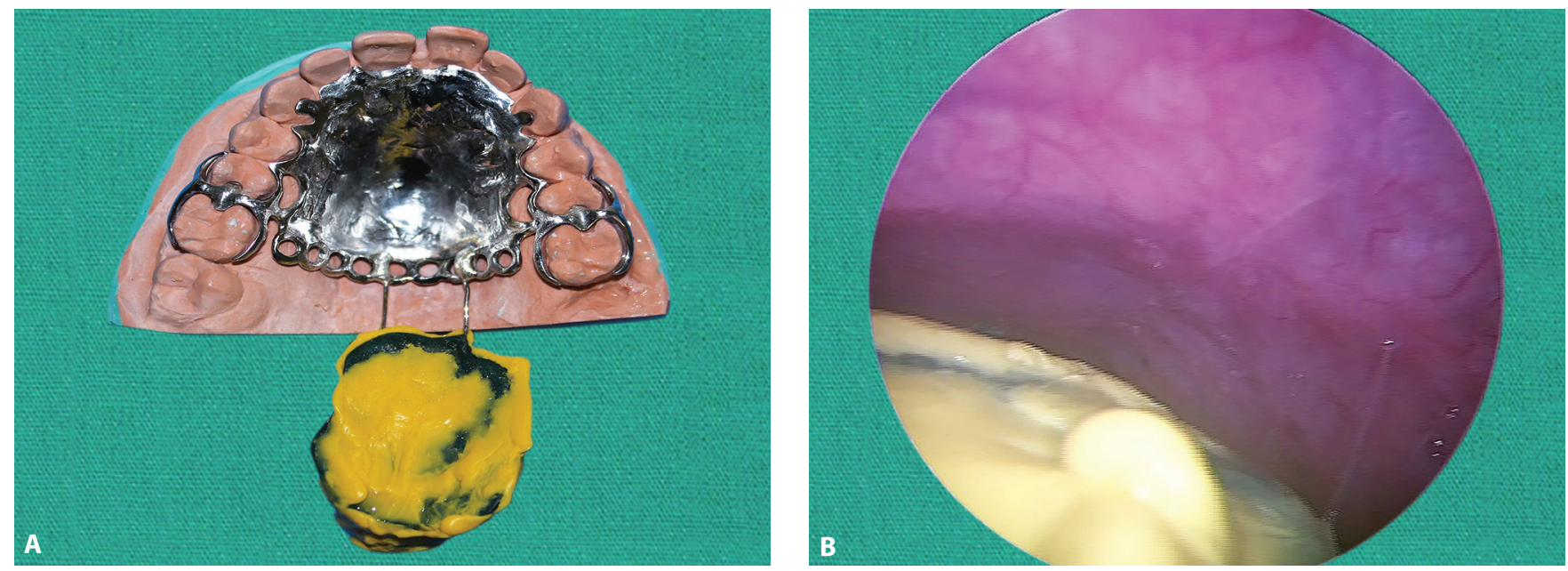

Figs 5A and B: (A) Border molding and final impression of defect area (B) Nasopharyngoscopic view

compound and the final impression was made using polyvinylsiloxane impression material of light body consistency (Fig. 5A). The accuracy of the impression and its close proximity with the posterior pharyngeal wall was checked using nasopharyngoscopy (Fig. 5B). The impression was repeated in case of any discrepancy. Then an altered cast technique was used and an altered master cast was poured to match with the difference in the resiliency of hard and soft tissues of the oral cavity. Then conventional lab procedures were followed such as wax-up, flasking, dewaxing and packing of the mold with the heat cured polymethylmethacrylate resin and cured using appropriate curing cycle. After finishing and polishing the final prosthesis was inserted in the patient mouth and a postoperative evaluation was done on visual and radiographical aspects.

Visual examination showed complete closure of the defect area (Fig. 6A) with clear speech and comprehensible sounds. Speech assessment and further improvement of speech was done with the help of a speech therapist. The radiographical evaluation showed the closure of posterior oropharyngeal space in lateral cephalogram and the close proximity of the prosthesis with the pharyngeal wall leaving a small space at the distal end which is required for the maintenance of normal respiration of the patient during rest and function in CBCT (Fig. 6B).

\section{Discussion}

Velopharyngeal (VP) defects were classified as VP insufficiency and VP incompetency. Main etiologies leading to VP insufficiency are congenital (cleft palate), trauma, developmental (short palate, deep nasopharynx) and surgical resection of neoplastic diseases. In this case, the etiology of VP insufficiency is secondary to surgical resection of pleomorphic adenoma of the minor salivary gland of soft palate. ${ }^{4}$

Pleomorphic adenoma is the most common benign tumor of salivary glands. Pleomorphic adenoma occurs in $45-75 \%$ of the total salivary gland tumors out of which most of the tumors occur in the parotid gland. But only in 5-10\% cases, it involved minor salivary glands. Most common sites for the involvement minor salivary glands are palate, lips, pharynx, trachea, and larynx. ${ }^{5}$ Surgical excision of the tumor remains the main line of treatment as it interferes during the normal function. Surgical excision of the tumor present in the soft palate leads to oro-nasal communication and VP insufficiency.

In normal VP closure, a definite seal is formed between the soft palate and posterior wall of pharynx leading to gushing of air through the oral cavity resulting in effective speech. But in VP insufficiency, this definite seal is not formed during speech and deglutition resulting 

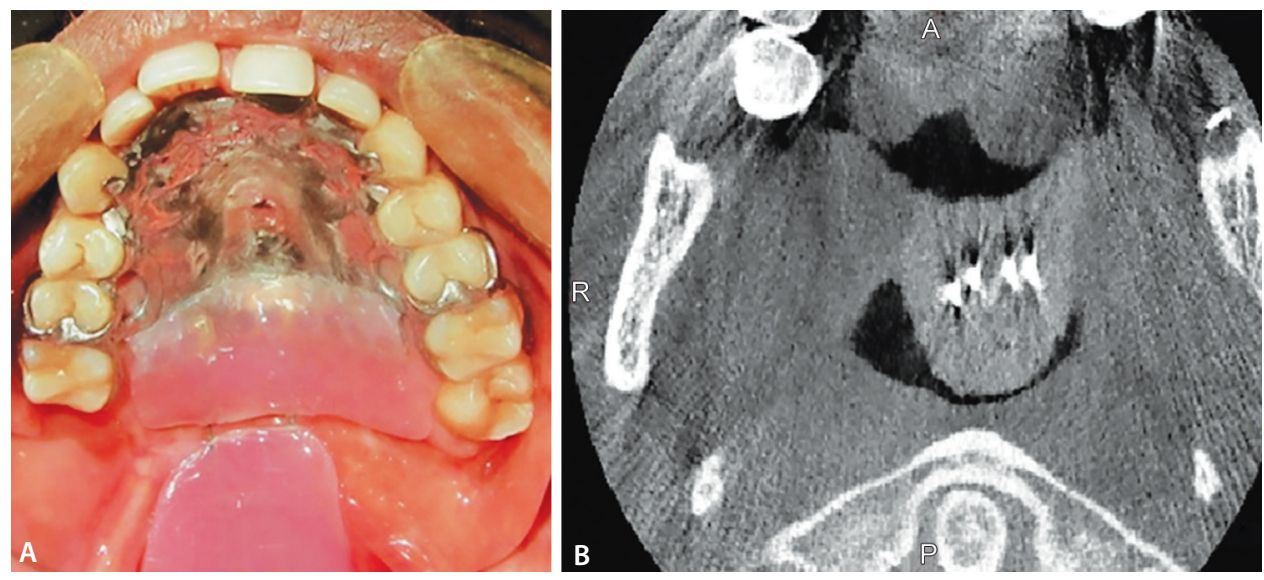

Figs 6 A and B: Postoperative (A) Intraoral (B) CBCT

in leakage of air through nasal cavity along with oral cavity adding hypernasality to voice resulting in inappropriate speech and leakage of food, fluids from nose. ${ }^{6}$

The multidisciplinary approach remains the mainstay of the management of patients with VP insufficiency. In this, an oncosurgeon/oral and maxillofacial surgeon, psychologist, ENT specialist, speech therapist, plastic surgeon, orthodontist, and prosthodontist plays a vital role. ${ }^{7}$

Various framework designs were used for various authors ranging from direct extension of cast metal framework in the defect area, ${ }^{8,9}$ use of a hinge at the distal end of prosthesis ${ }^{10}$ and use of wire extension in zigzag manner. ${ }^{11}$ In this case, a 21 gauge orthodontic wire was soldered to the distal end of the metal framework and extending to the defect area which provides adequate strength as well as the flexibility to the distal end so that prosthesis acts an integral part of the soft palate during different functional movements.

Nasopharyngoscopy serves as a definitive aid for the evaluation of VP function. It is used to evaluate the accuracy of the prosthesis and its relationship with the posterior pharyngeal wall in rest and during the function. It allows direct visualization of a nasopharyngeal sphincter without interfering with speech. ${ }^{12,13}$ However, nowadays, CBCT is also used in the assessment of pharyngeal airway space and therefore helpful in the evaluation of the accuracy of the prosthesis in conditions of unavailability of nasopharyngoscopic unit. ${ }^{14}$

\section{CONCLUSION}

Velopharyngeal (VP) defects are complex conditions as patients have problems such as inappropriate speech and regurgitation of food. Improvement of quality of life of such patients is of utmost importance. They have freedom of speech, but they are not able to speak which creates a psychological effect on the patient. So a holistic and multidisciplinary approach is required which will provide excellent results and improve the quality of life of the patient.

\section{References}

1. Hobkirk Z, Jacob E. Prosthodontic treatment for edentulous patient: Complete dentures and implant-supported prostheses. 13th Edition; 2004:351-354.

2. Abreu A, Levy D, Rodriguez E, et al. Oral rehabilitation of a patient with complete unilateral cleft lip and palate using an implantretained speech-aid prosthesis: Clinical report. Cleft Palate Craniofac J.2007;44:673-677.

3. Shifman A, Finkelstein $Y$, Nachmani A, et al. Speech-aid prostheses for neurogenic velopharyngeal incompetence. J Prosthet Dent. 2000;83:99-106.

4. Shetty NB, Shetty S, Nagraj E.,et al. Management of velopharyngeal defects: A Review. J Clin Diagn Res. 2014; 8(3):283-287.

5. Khan MN, Raza SS, Hussain Zaidi SA, et al. Pleomorphic adenoma of minor salivary glands.J Ayub Med Coll Abbottabad.2016;28(3):620-622.

6. Perry JL. Anatomy and physiology of the velopharyngeal mechanism. Semin Speech Lang.2011;32(2):83-92.

7. Rieger J, Bohle lii G, Huryn J, et al. Surgical reconstruction versus prosthetic obturation of extensive soft palate defects: a comparison of speech outcomes. Int J Prosthodont. 2009;22:566572.

8. Gupta SH, Viswambaran M, Vijayakumar R. Management of a case of velopharyngeal insufficiency with multidisciplinary approach. Med J Armed Forces India.2015;71(Suppl 2):S521-S524.

9. Banerjee S, Kumar S, Chakraborty N, et al. Prosthodontic rehabilitation of velopharyngeal disorders-A case series. J Indian Prosthodont Soc. 2013;13(3): 352-357.

10. Ram HK, Shah RJ. A novel approach for velopharyngeal prosthetic rehabilitation: Case series.Int J Healthcare Biomed Res.2013:1(2):7076.

11. Uphadhyay M, Jain D, Kumar S, et al. Speech aid prosthesis. BMJ Case Rep. 2013; 2013:1-3.

12. D'Antonio LL, Muntz HR, Marsh JL, et al. Practical application of flexible fiberoptic nasopharyngoscopy for evaluating velopharyngeal function. Plast Reconstr Surg. 1988;82(4):611-618.

13. Rieger JM, Zalmanowitz JG, Wolfaardt JF. Nasopharyngoscopy in palatopharyngeal prosthetic rehabilitation: A preliminary report. Int J Prosthodont.2006;19(4):383-388.

14. Hatcher DC. Cone beam computed tomography: craniofacial and airway analysis. Dent Clin North Am. 2012;56(2):343-357. 